\title{
TWO APPLICATIONS OF ASYMPTOTIC PRIME DIVISORS
}

\author{
STEPHEN MCADAM ${ }^{1}$
}

\begin{abstract}
Some recent interest has focused on the set of prime divisors of large powers of an ideal in a Noetherian ring. This note presents two results whose proofs appear to depend on knowledge of such asymptotic prime divisors.
\end{abstract}

Introduction. Let $I$ be an ideal in a Noetherian ring $R$. It was recently shown that, for all large $n, \operatorname{Ass}\left(R / I^{n}\right)=\operatorname{Ass}\left(R / I^{n+1}\right)$ [1]. Many interesting ideas have ensued. For example, we prove the following two results.

THEOREM A. Let $\bar{R}$ be the integral closure of the Noetherian domain $R$. If $J$ is a finitely generated ideal of $\bar{R}$, then only finitely many primes of $\bar{R}$ are minimal over $J$.

THeOREM B. Let $R \subseteq T$ be an integral extension of domains with $R$ Noetherian. If $Q$ is prime in $T$ and height $Q=n$, then grade $Q \cap R \leqslant n$. Furthermore, if grade $Q \cap R=n$, then $Q \cap R$ is a prime divisor of any ideal generated by a maximal $R$-sequence from $Q \cap R$.

Needing only a fraction of the existing knowledge of asymptotic prime divisors, we present it, rather than just giving references.

Lemma [5]. Let $I$ be an ideal in a Noetherian ring $R$. The set $\cup \operatorname{Ass}\left(R / I^{n}\right)$, $n=1,2, \ldots$, is finite.

Proof. Let $t$ be an indeterminate and let $A=R\left[t^{-1}, I t\right]$, the Rees ring. Now $t^{-n} A \cap R=I^{n}$, and if $P \in \operatorname{Ass}\left(R / I^{n}\right)$ one easily finds $Q \in \operatorname{Ass}\left(A / t^{-n} A\right)$ with $Q \cap R=P$. As $t^{-1}$ is regular, $Q \in \operatorname{Ass}\left(A / t^{-1} A\right)$, which is a finite set.

Lemma [3]. Let $R \subset T$ be an integral extension of domains, $R$ Noetherian. Let I be an ideal of $R$ and let $Q \in \operatorname{Spec} T$ with $Q$ minimal over $I T$. Then $P=Q \cap R \in$ $\cup \operatorname{Ass}\left(R / I^{n}\right)$.

Proof. We may assume $R$ is local at $P$. We also assume $T=R[u]$ with $u \in \bar{R}$. To do this, by going up assume $T=\bar{T}$, and then by going down assume $T=\bar{R}$. Finally, choose $u \in Q$ but in no other prime of $\bar{R}$ lying over $P$. Thus only $Q$ lies over $Q \cap R[u]$, and so we assume $T=R[u]$.

\footnotetext{
Received by the editors March 16, 1981 and, in revised form, May 19, 1981.

1980 Mathematics Subject Classification. Primary 13E05.

Key words and phrases. Noetherian domain, prime divisor, asymptotic prime divisor, integral exten-

${ }^{1}$ Partially supported by the National Science Foundation.
} sion. 
Pick $0 \neq b \in R$ with $b T \subseteq R$, and $n$ large enough that $b \notin Q^{n}$. As $Q$ is minimal over $I^{n} T$, there is a $k>0$ and an $s \in T-Q$ with $s Q^{k} \subseteq I^{n} T$. Thus $b s P^{k} \subseteq b s Q^{k}$ $\subseteq b s I^{n} T \subseteq I^{n}$, since $b T \subseteq R$. However $b s \in R-I^{n}$, since if $b s \in I^{n} \subseteq Q^{n}$, then since $Q^{n}$ is primary to the maximal $Q, b \in Q^{n}$ a contradiction. Therefore $P^{k}$ consists of zero divisors modulo $I^{n}$, and being maximal, $P \in \operatorname{Ass}\left(R / I^{n}\right)$.

Proof of TheOrem A. Let $J=\left(a_{1}, \ldots, a_{m}\right) \bar{R}$. Let $R_{1}=R\left[a_{1}, \ldots, a_{m}\right]$ and $I=\left(a_{1}, \ldots, a_{m}\right) R_{1}$. Since $J=I \bar{R}$, if $Q \in \operatorname{Spec} \bar{R}$ and $Q$ is minimal over $J$, then $Q \cap R \in \cup \operatorname{Ass}\left(R_{1} / I^{n} R_{1}\right)$. The first lemma, and the fact that only finitely many primes of $\bar{R}$ lie over any prime of $R_{1}$, give the result.

Proof of Theorem B. Induct on $n$. If $n=1$, pick $0 \neq a \in Q \cap R$. Thus $Q$ is minimal over $a T$, so $Q \cap R \in \operatorname{Ass}\left(R / a^{m} R\right)$, some $m$. Therefore $Q \cap R \in$ $\operatorname{Ass}(R / a R)$. For $n>1$, suppose grade $Q \cap R>n-1$ and let $a_{1}, \ldots, a_{n}$ be an $R$-sequence from $Q \cap R$. By induction, we see that height $\left(a_{1}, \ldots, a_{n}\right) T \geqslant n$. Thus $Q$ is minimal over $\left(a_{1}, \ldots, a_{n}\right) T$ so that $Q \cap R$ is a prime divisor of $\left(a_{1} R, \ldots, a_{n} R\right)^{m}$, some $m$. As $a_{1}, \ldots, a_{n}$ is an $R$-sequence, $Q \cap R$ is also a prime divisor of $\left(a_{1}, \ldots, a_{n}\right) R[2, \$ 3.1$, Exercise 13].

Theorem B extends $[4,33.11]$.

ADDED IN PROOF. A recently discovered sophisticated argument shows that in Theorem B, height $Q=n$ can be weakened to little height $Q=n$.

\section{REFERENCES}

1. M. Brodmann, Asymptotic stability of Ass $\left(R / I^{n}\right)$, Proc. Amer. Math. Soc. (to appear).

2. I. Kaplansky, Commutative rings, Univ. of Chicago Press, Chicago, Ill., 1974.

3. S. McAdam, Asymptotic prime divisors and going down, Pacific J. Math. 91 (1980), 179-186.

4. M. Nagata, Local rings, Interscience, New York, 1962.

5. L. J. Ratliff, Jr., On prime divisors of $I^{n}$, n large, Michigan Math. J. 23 (1976), 337-352.

Department of Mathematics, University of Texas, Austin, TeXas 78712 